\title{
A challenging diagnosis of dyspnea: A case report of contralateral reexpansion pulmonary edema
}

\author{
Alfonso Sforza, Maria V. Carlino, Giovanni Albano, Maria I. Arnone, Giuliano De Stefano, \\ Andrea D’Amato, Federica De Pisapia, Giovanni de Simone, Costantino Mancusi \\ Hypertension Research Center, UOC Emergency Medicine, Federico II University Hospital, Naples, Italy
}

\begin{abstract}
Reexpansion pulmonary edema (RPE) is an uncommon complication of thoracentesis or chest drainage. It occurs in the ipsilateral or contralateral lung. Causes, pathogenesis and therapy are not well understood especially for contralateral RPE. We describe a case of fatal contralateral RPE in a 59-years-old woman with right lung cancer underwent ultrasound-guided thoracentesis for massive pleural effusion and severe dyspnea. Pathogenesis of contralateral RPE is probably multifactorial and in this case is mostly due to the overperfusion of the healthy lung and consequent capillary damage. The right therapy for this condition is not known.
\end{abstract}

\section{Introduction}

Reexpansion pulmonary edema (RPE) is an uncommon lifethreatening complication of removing air or fluid from the pleural cavity by either thoracentesis or chest drainage. It occurs in the af-

Corresponding author: Dr. Costantino Mancusi, Hypertension Research Center, University of Naples Federico II, Via Pansini 5, bld \#1, 80131 Napoli, Italy. Tel. +39.081.7462025. E-mail: costantino.mancusi@unina.it

Key words: Thoracentesis; lung ultrasound; point-of-care ultrasound; acute dyspnea; lung cancer.

Contributions: AS, MVC, conceived the paper; AS, GA, wrote the manuscript; CM, MIA, GDS, ADA, FDP, GdS contributed to the discussion and edited the manuscript.

Conflict of interest: The authors declare no conflict of interest.

Received for publication: 12 December 2017

Accepted for publication: 4 March 2018

(C) Copyright A. Sforza et al., 2018

Tipografia PI-ME Editrice, Italy

Monaldi Archives for Chest Disease 2018; 88:900

doi: 10.4081/monaldi.2018.900

This article is distributed under the terms of the Creative Commons Attribution Noncommercial License (by-nc 4.0) which permits any noncommercial use, distribution, and reproduction in any medium, provided the original author(s) and source are credited. fected lung but rarely also in the contralateral lung. Causes, pathogenesis and therapy are not well understood especially for contralateral RPE.

\section{Case Report}

A 59-years-old woman with history of end-stage right lung cancer was admitted to the emergency department for worsening dyspnea during last days. She received diagnosis of squamous cell lung cancer three years before and she underwent full oncological treatment. She was current smoker with chronic obstructive pulmonary disease. She was on therapy with corticosteroids and opioids. The last CT scan performed three weeks before revealed progression of the lung cancer with concomitant development of right side pleural effusion.

On admission she was conscious with no airway obstruction, oxygen saturation was $75 \%$ on room air with tachypnea (35 breaths per min). Blood pressure (BP) was $90 / 60 \mathrm{mmHg}$, heart rate (HR) was 110 bmp regular and she was afebrile. Chest examination revealed abolished vesicular murmur in the right side and normal sounds in the left, cardiovascular examination revealed normal heart sounds, peripheral pulses were palpable but weak, abdominal exam was normal. There was no focal neurological deficit and there were signs of dehydration. Electrocardiogram showed sinus tachycardia. Arterial blood gas analysis on room air revealed prevalent respiratory alkalosis with mild metabolic acidosis and severe hypoxia, normal hemoglobin and electrolytes, increased lactate levels (Table 1). Chest X-ray showed massive right pleural effusion and atelectasis of lower and median lobes (Figure 1). Multi-organ ultrasound [1] showed normal cardiac structure and function, massive pleural effusion in the right side (Figure 2) and A-profile in the left lung. The patient received oxygen therapy with Venturi mask $\left(\mathrm{FiO}_{2} 50 \%\right)$ with improvement of saturation and mild reduction of tachypnea $\left(\mathrm{SaO}_{2} 94 \%\right.$, respiratory rate (RR) 30 breaths/min). Ultrasound-guided thoracentesis was performed on the right side resulting in slow aspiration of $1000 \mathrm{ml}$ of citrine fluid with consequent improvement of symptoms and vital signs (BP 110/70 mmHg, HR 90 bmp, RR 20 breaths/min, $\mathrm{SaO}_{2} 96 \%$ with nasal cannula $4 \mathrm{~L} / \mathrm{min}$ ). During the procedure, she received $500 \mathrm{ml}$ of normal saline and a corticosteroid therapy with $40 \mathrm{mg}$ of metilprednisolone was started. The day after the procedure patient complained sudden intense dyspnea with diffuse crackles in the left lung. Chest X-ray showed interstitial edema on the left side, lung ultrasound showed white left lung (Figure 3 ) and moderate pleural effusion with partial atelectasis on the right lung confirming the diagnosis of contralateral RPE. She was placed on continuous positive airway pressure and treated with furosemide, nitrates and morphine with partial benefits. The patient's condition worsened progressively and she died in few hours. 


\section{Discussion}

Pathogenesis of RPE is probably multifactorial. The pathophysiologic mechanism proposed for the ipsilateral RPE is the damage of pulmonary microvessels with loss of integrity of the alveolar-capillary membrane due to anoxic stress during lung collapse and stretching injury during re-expansion. Moreover, the reperfusion of the previously not-ventilated and not-perfused lung can cause the production of oxygen-derived free radicals and inflammatory mediators resulting in inflammatory injury. The decrease of alveolar surfactant activity can also be involved [2]. Rapid re-expansion, drainage with the use of negative intrapleural pressure and chronicity of lung collapse are considered the major risk factors for reexpansion pulmonary edema [3]. The contralateral RPE is less understood. Possible hypothesis for its pathogenesis are oblivious aspiration, systemic inflammatory response after reexpansion in case of significant lung disease, significant increased cardiac output after rapid reexpansion of the lung [4]. In this case we believe that the most important cause of the contralateral RPE is the over-perfusion of the healthy lung. The reexpansion with reperfusion of a partial not-ventilated lung (due to atelectasis from cancer bronchial obstruction) causes intrapulmonary shunting that produces local hypoxemia and consequent vasoconstriction of the ipsilateral pulmonary arterial field [5]. This redirects blood flow to the contralateral lung with over-perfusion and consequent cap-

Table 1. Arterial blood gas analysis on admission.

\begin{tabular}{lcc} 
Variables & Values & Reference ranges \\
$\mathrm{pH}$ & 7.46 & $7.38-7.42$ \\
\hline $\mathrm{pCO}_{2}(\mathrm{mmHg})$ & 22.6 & $38-42$ \\
\hline $\mathrm{pO}_{2}(\mathrm{mmHg})$ & 41 & $85-98$ \\
\hline $\mathrm{HCO}_{3}-(\mathrm{mEq} / \mathrm{L})$ & 15.6 & $24-26$ \\
\hline Lact $(\mathrm{mmol} / \mathrm{L})$ & 2.5 & $<1.6$ \\
\hline
\end{tabular}

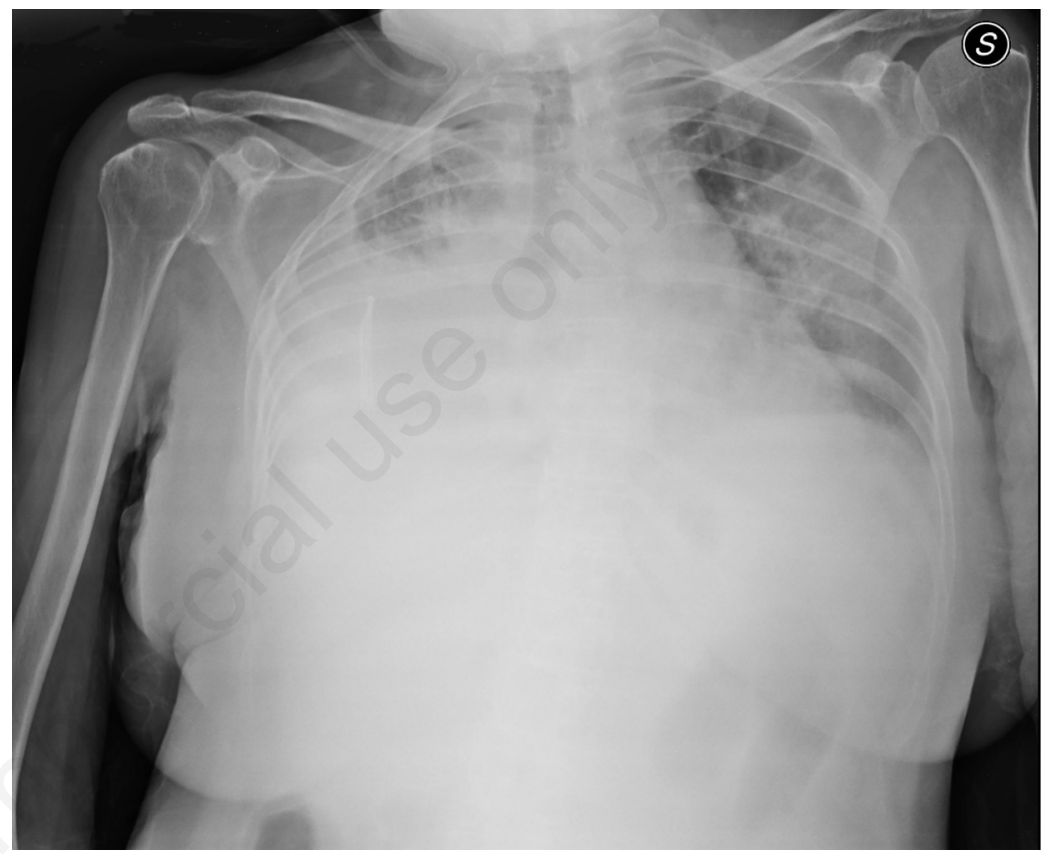

Figure 1. Chest X-ray: massive right pleural effusion. illary damage. The implication of the inflammatory injury mechanism was probably less relevant cause the patient was on steroid therapy that should have led to a reduction in the inflammatory response.

\section{Conclusions}

Contralateral RPE is a rare and life-threatening condition that can occur when a collapsed lung is re-expanded. Since this condition probably results from both fluid overload and inflammatory damage of the healthy lung, the therapy has to take into account diuretics, corticosteroids and ventilatory support.

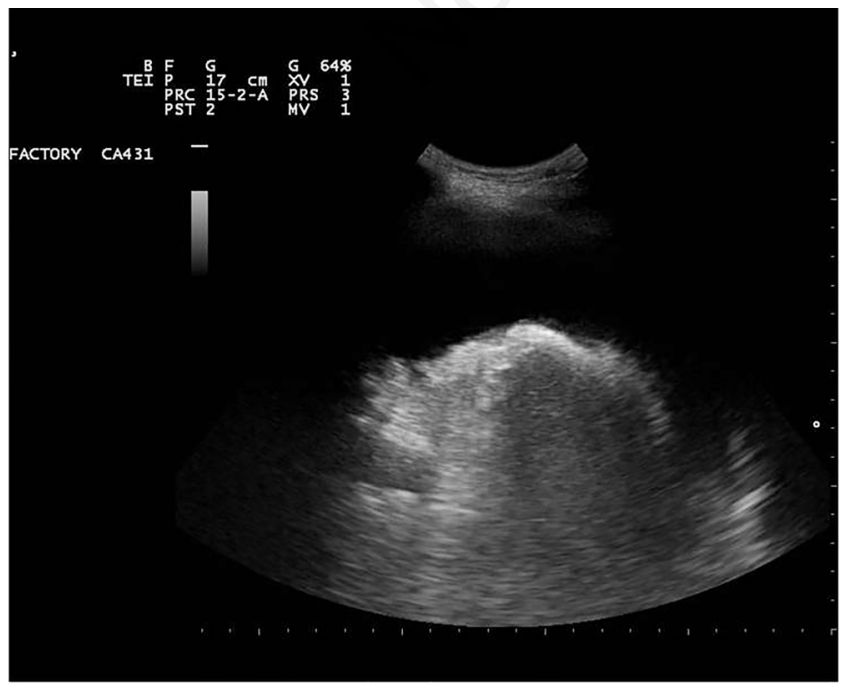

Figure 2. Lung ultrasound: right pleural effusion. 


\section{References}

1. Sforza A, Mancusi C, Carlino MV, et al. Diagnostic performance of multi-organ ultrasound with pocket-sized device in the management of acute dyspnea. Cardiovasc Ultrasound 2017;15:16.

2. Sohara Y. Reexpansion pulmonary edema. Ann Thorac Cardiovasc Surg 2008;14:205-9.
3. Yoon JS, Suh JH, Choi SY, et al. Risk factors for the development of reexpansion pulmonary edema in patients with spontaneous pneumothorax. J Cardiothorac Surg 2013;8:164.

4. Kim JJ, Kim YH, Choi SY et al. Contralateral reexpansion pulmonary edema with ipsilateral collapsed lung after pleural effusion drainage: a case report. J Cardiothorac Surg 2015;10:68.

5. Ferreira HC, Zin WA, Macedo Rocco PR. Physiopathology and clinical management of one-lung ventilation. J Bras Pneumol 2004;30:566-73. 